\title{
The tangent spherical image and ccr-curve of a time-like curve in $\mathbb{L}^{3}$
}

\section{Esen İyigün*}

\section{*Correspondence:}

esen@uludag.edu.tr

Department of Mathematics, Art and Science Faculty, Uludağ

University, Bursa, 16059, Turkey

\begin{abstract}
In this work, we define the tangent spherical image of a unit speed time-like curve lying on the pseudohyperbolic space $H_{0}^{2}(r)$ in $\mathbb{L}^{3}$. In addition, we calculate a ccr-curve of this curve in $\mathbb{L}^{3}$. Besides, we determine a relation between harmonic curvature and a ccr-curve in $\mathbb{L}^{3}$, and so we obtain some new results.
\end{abstract}

\section{Introduction}

Let $X=\left(x_{1}, x_{2}, x_{3}\right)$ and $Y=\left(y_{1}, y_{2}, y_{3}\right)$ be two non-zero vectors in the three-dimensional Lorentz-Minkowski space $\mathbb{R}_{1}^{3}$. We denoted $\mathbb{R}_{1}^{3}$ shortly by $\mathbb{L}^{3}$. For $X, Y \in \mathbb{L}^{3}$,

$$
\langle X, Y\rangle=-x_{1} y_{1}+x_{2} y_{2}+x_{3} y_{3}
$$

is called a Lorentzian inner product. The couple $\left\{\mathbb{R}_{1}^{3},\langle\rangle,\right\}$ is called a Lorentzian space and denoted by $\mathbb{L}^{3}$. Then the vector $X$ of $\mathbb{L}^{3}$ is called

(i) time-like if $\langle X, X\rangle<0$,

(ii) space-like if $\langle X, X\rangle>0$ or $X=0$,

(iii) a null (or light-like) vector if $\langle X, X\rangle=0, X \neq 0$.

The norm of a vector $X$ is given by $\|X\|=\sqrt{|\langle X, X\rangle|}$. Therefore, $X$ is a unit vector if $\langle X, X\rangle= \pm 1$. Next, vectors $X, Y$ in $\mathbb{L}^{3}$ are said to be orthogonal if $\langle X, Y\rangle=0$. The velocity of a curve $\alpha(s)$ is given by $\left\|\alpha^{\prime}(s)\right\|$. Space-like or time-like $\alpha(s)$ is said to be parametrized by an arclength function $s$ if $\left\langle\alpha^{\prime}(s), \alpha^{\prime}(s)\right\rangle= \pm 1$ [1]. For any $X=\left(x_{1}, x_{2}, x_{3}\right), Y=\left(y_{1}, y_{2}, y_{3}\right) \in \mathbb{R}_{1}^{3}$, the pseudo-vector product of a $X$ and $Y$ is defined as follows:

$$
X \Lambda Y=\left(-\left(x_{2} y_{3}-x_{3} y_{2}\right), x_{3} y_{1}-x_{1} y_{3}, x_{1} y_{2}-x_{2} y_{1}\right)
$$

[2].

\section{Basic concepts}

Definition 1.1 An arbitrary curve $\alpha: I \rightarrow \mathbb{L}^{3}$ in the space $\mathbb{L}^{3}$ can locally be space-like, time-like or a null curve if, respectively, all of its velocity vectors $\alpha^{\prime}(s)$ are space-like, timelike or null [3].

Definition 1.2 Let $\alpha \subset \mathbb{L}^{3}$ be a given time-like curve. If the Frenet vector $\left\{V_{1}(s), V_{2}(s)\right.$, $\left.V_{3}(s)\right\}$ which corresponds to $s \in I$ is defined as

$$
k_{i}: I \rightarrow \mathbb{R}, \quad k_{i}(s)=\left\langle V_{i}^{\prime}(s), V_{i+1}(s)\right\rangle
$$


then the function $k_{i}$ is called an $i$ th curvature function of the time-like curve $\alpha$, and the real $k_{i}(s)$ is also called an $i$ th curvature at the point $\alpha(s)$ [4].

Definition 1.3 Let $\alpha: I \longrightarrow \mathbb{L}^{3}$ be a unit speed non-null curve in $\mathbb{L}^{3}$. The curve $\alpha$ is called a Frenet curve of osculating order $d(d \leq 3)$ if its 3rd order derivatives $\alpha^{\prime}(s), \alpha^{\prime \prime}(s), \alpha^{\prime \prime \prime}(s)$ are linearly independent and $\alpha^{\prime}(s), \alpha^{\prime \prime}(s), \alpha^{\prime \prime \prime}(s), \alpha^{l v}(s)$ are no longer linearly independent for all $s \in I$. For each Frenet curve of order 3, one can associate an orthonormal 3-frame $\left\{V_{1}(s), V_{2}(s), V_{3}(s)\right\}$ along $\alpha$ (such that $\left.\alpha^{\prime}(s)=V_{1}\right)$ called the Frenet frame and $k_{1}, k_{2}: I \rightarrow \mathbb{R}$ called the Frenet curvatures, such that the Frenet formulas are defined in the usual way:

$$
\begin{aligned}
& V_{1}^{\prime}=\nabla_{\nu_{1}} \alpha^{\prime}=\varepsilon_{2} k_{1} V_{2}, \\
& V_{2}^{\prime}=\nabla_{\nu_{1}} V_{2}=-\varepsilon_{1} k_{1} V_{1}+\varepsilon_{3} k_{2} V_{3}, \\
& V_{3}^{\prime}=\nabla_{\nu_{1}} V_{3}=-\varepsilon_{2} k_{2} V_{2},
\end{aligned}
$$

where $\nabla$ is the Levi-Civita connection of $\mathbb{L}^{3}$.

Definition 1.4 A non-null curve $\alpha: I \longrightarrow \mathbb{L}^{3}$ is called a $W$-curve (or helix) of rank 3, if $\alpha$ is a Frenet curve of osculating order 3 and the Frenet curvatures $k_{i}, 1 \leq i \leq 2$, are non-zero constants.

\section{Harmonic curvatures and constant curvature ratios in $\mathbb{L}^{3}$}

Definition 2.1 Let $\alpha$ be a non-null curve of osculating order 3. The harmonic functions

$$
H_{j}: I \rightarrow \mathbb{R}, \quad 0 \leq j \leq 1,
$$

defined by

$$
\left\{\begin{array}{l}
H_{0}=0, \\
H_{1}=\frac{k_{1}}{k_{2}}
\end{array}\right.
$$

are called harmonic curvatures of $\alpha$, where $k_{1}, k_{2}$ are Frenet curvatures of $\alpha$ which are not necessarily constant.

Definition 2.2 Let $\alpha$ be a time-like curve in $\mathbb{L}^{3}$ with $\alpha^{\prime}(s)=V_{1} . X \epsilon \chi\left(\mathbb{L}^{3}\right)$ being a constant unit vector field, if

$$
\left\langle V_{1}, X\right\rangle=\cosh \varphi \quad \text { (constant) }
$$

then $\alpha$ is called a general helix (inclined curves) in $\mathbb{L}^{3}, \varphi$ is called a slope angle and the space $\operatorname{Sp}\{X\}$ is called a slope axis [5].

Definition 2.3 Let $\alpha$ be a non-null of osculating order 3. Then $\alpha$ is called a general helix of rank 1 if

$$
H_{1}^{2}=c
$$

holds, where $c \neq 0$ is a real constant. 
We have the following results.

\section{Corollary 2.1}

(i) If $H_{1}=0$, then $\alpha$ is a straight line.

(ii) If $H_{1}$ is constant, then $\alpha$ is a general helix of rank 1.

Proof By the use of the above definition, we obtain the proof.

Proposition 2.1 Let $\alpha$ be a curve in $\mathbb{L}^{3}$ of osculating order 3 . Then

$$
\begin{aligned}
& V_{1}^{\prime}=\varepsilon_{2} k_{2} H_{1} V_{2}, \\
& V_{2}^{\prime}=-\varepsilon_{1} k_{2} H_{1} V_{1}+\varepsilon_{3} \frac{k_{1}}{H_{1}} V_{3}, \\
& V_{3}^{\prime}=-\varepsilon_{2} \frac{k_{1}}{H_{1}} V_{2},
\end{aligned}
$$

where $H_{1}$ is harmonic curvature of $\alpha$.

Proof By using the Frenet formulas and the definitions of harmonic curvatures, we get the result.

Now, we will give the relation between harmonic curvature and a ccr-curve in $\mathbb{L}^{3}$.

Definition 2.4 A curve $\alpha: I \longrightarrow \mathbb{L}^{3}$ is said to have constant curvature ratios (that is to say, it is a ccr-curve) if all the quotients $\varepsilon_{i}\left(\frac{k_{i+1}}{k_{i}}\right)$ are constant, where $\varepsilon_{i}=\left\langle V_{i}, V_{i}\right\rangle= \pm 1$.

Corollary 2.2 For $i=1$, the ccr-curve is $\frac{\varepsilon_{1}}{H_{1}}$.

Proof The proof can be easily seen by using the definitions of harmonic curvature and ccr-curve.

Corollary 2.3 Let $\alpha: I \longrightarrow \mathbb{L}^{3}$ be a ccr-curve. If $\frac{\varepsilon_{1}}{H_{1}}=c$, c is a constant, then $\left(\frac{\varepsilon_{1}}{H_{1}}\right)^{\prime}=0$.

Proof The proof is obvious.

\section{Tangent spherical image}

Definition 3.1 [1] Let $n \geq 2$ and $0 \leq v \leq n$. Then the pseudohyperbolic space of radius $r>0$ in $\mathbb{R}_{1}^{3}$ is the hyperquadric

$$
H_{0}^{2}(r)=\left\{p \in \mathbb{R}_{1}^{3}:\langle p, p\rangle=-r^{2}\right\}
$$

with dimension 2 and index 0 .

Definition 3.2 Let $\alpha=\alpha(s)$ be a unit speed time-like curve in $\mathbb{L}^{3}$. If we translate the tangent vector to the center 0 of the pseudohyperbolic space $H_{0}^{2}(r)$, we obtain a curve $\delta=\delta\left(s_{\delta}\right)$. This curve is called the tangent spherical image of a curve $\alpha$ in $\mathbb{L}^{3}$. 


\section{Theorem 3.1 [6]}

(i) Let $\alpha=\alpha(s)$ be a unit speed time-like curve and $\delta=\delta\left(s_{\delta}\right)$ be its tangent spherical image. Then $\delta=\delta\left(s_{\delta}\right)$ is a space-like curve.

(ii) Let $\alpha=\alpha(s)$ be a unit speed time-like curve and $\delta=\delta\left(s_{\delta}\right)$ be its tangent spherical image. If $\alpha$ is a ccr-curve or a helix (i.e. $W$-curve), then $\delta$ is also a helix.

Proof From [6] it is easy to see the proof of the theorem.

\section{An example}

Example 4.1 Let us consider the following curve in the space $\mathbb{L}^{3}$ :

$$
\begin{aligned}
& \alpha(s)=(\sqrt{2} s, \cos s, \sin s), \\
& V_{1}(s)=\alpha^{\prime}(s)=(\sqrt{2},-\sin s, \cos s),
\end{aligned}
$$

where $\left\langle\alpha^{\prime}(s), \alpha^{\prime}(s)\right\rangle=-1$, which shows $\alpha(s)$ is a unit speed time-like curve. Thus $\left\|\alpha^{\prime}(s)\right\|=1$. We express the following differentiations:

$$
\begin{aligned}
& \alpha^{\prime \prime}(s)=(0,-\cos s,-\sin s), \\
& \Rightarrow \quad \alpha^{\prime \prime \prime}(s)=(0, \sin s,-\cos s)
\end{aligned}
$$

and

$$
V_{2}(s)=\frac{\alpha^{\prime \prime}(s)}{\left\|\alpha^{\prime \prime}(s)\right\|}=\alpha^{\prime \prime}(s)
$$

So, we have the first curvature as

$$
k_{1}(s)=\left\langle V_{1}^{\prime}(s), V_{2}(s)\right\rangle=1=\text { constant }
$$

Moreover, we can write the third Frenet vector of the curve as follows:

$$
V_{3}(s)=V_{1}(s) \Lambda V_{2}(s)=(-1, \sqrt{2} \sin s,-\sqrt{2} \cos s)
$$

Finally, we have the second curvature of $\alpha(s)$ as

$$
k_{2}(s)=\left\langle V_{2}^{\prime}(s), V_{3}(s)\right\rangle=\sqrt{2}=\text { constant }
$$

Now, we will calculate a ccr-curve of $\alpha(s)$ in $\mathbb{L}^{3}$. If the vector $V_{1}$ is time-like, then $\varepsilon_{1}=-1$,

$$
\varepsilon_{1} \frac{k_{2}}{k_{1}}=-\sqrt{2}=\text { constant }
$$

Thus $\alpha(s)$ is a ccr-curve in $\mathbb{L}^{3}$. 
Acknowledgements

Dedicated to Prof. Hari M. Srivastava.

Received: 11 December 2012 Accepted: 28 January 2013 Published: 18 February 2013

\section{References}

1. O'Neill, B: Semi-Riemannian Geometry with Applications to Relativity. Academic Press, New York (1983)

2. Öğrenmiş, AO, Balgetir, H, Ergüt, M: On the ruled surfaces in Minkowski 3-space $\mathbb{R}_{1}^{3}$. J. Zhejiang Univ. Sci. A 7(3), 326-329 (2006)

3. Petrovic-Torgasev, M, Sucurovic, E: W-curves in Minkowski space-time. Novi Sad J. Math. 32(2), 55-65 (2002)

4. Bektaş, M, Ergüt, M, Soylu, D: The characterization of the spherical timelike curves in 3-dimensional Lorentzian space. Bull. Malays. Math. Soc. 21, 117-125 (1998)

5. Ekmekçi, N, Hacisalihoglu, HH, Illarslan, K: Harmonic curvatures in Lorentzian space. Bull. Malays. Math. Soc. 23 173-179 (2000)

6. Yılmaz, S, Özyılmaz, E, Yaylı, Y, Turgut, M: Tangent and trinormal spherical images of a time-like curve on the pseudohyperbolic space $H_{0}^{3}$. Proc. Est. Acad. Sci. 59(3), 216-224 (2010)

doi:10.1186/1029-242X-2013-55

Cite this article as: İyigün: The tangent spherical image and ccr-curve of a time-like curve in $\mathbb{L}^{3}$. Journal of Inequalities and Applications 2013 2013:55.

\section{Submit your manuscript to a SpringerOpen ${ }^{\circ}$ journal and benefit from:}

- Convenient online submission

- Rigorous peer review

Immediate publication on acceptance

- Open access: articles freely available online

- High visibility within the field

- Retaining the copyright to your article 